\title{
automemcpy: A Framework for Automatic Generation of Fundamental Memory Operations*
}

\author{
Guillaume Chatelet \\ Google Research \\ France \\ gchatelet@google.com \\ Ondrej Sykora \\ Google Research \\ France \\ ondrasej@google.com
}

\author{
Chris Kennelly \\ Google \\ USA \\ ckennelly@google.com \\ Clément Courbet \\ Google Research \\ France \\ courbet@google.com
Bruno De Backer
Google Research
France
bdb@google.com

\author{
Sam (Likun) Xi \\ Google \\ USA \\ xyzsam@google.com \\ Xinliang David Li \\ Google \\ USA \\ davidxl@google.com
}

\begin{abstract}
Memory manipulation primitives (memcpy, memset, memcmp) are used by virtually every application, from high performance computing to user interfaces. They often consume a significant portion of CPU cycles. Because they are so ubiquitous and critical, they are provided by language runtimes and in particular by $l i b c$, the $\mathrm{C}$ standard library. These implementations are heavily optimized, typically written in hand-tuned assembly for each target architecture.

In this article, we propose a principled alternative to handtuning these functions: (1) we profile the calls to these functions in their production environment and use this data to drive the important high-level algorithmic decisions, (2) we use a high-level language for the implementation, delegate the job of tuning the generated code to the compiler, and (3) we use constraint programming and automatic benchmarks to select the optimal high-level structure of the functions.

We compile our memfunctions implementations using the same compiler toolchain that we use for application code, which allows leveraging the compiler further by allowing whole-program optimization. We have evaluated our approach by applying it to the fleet of one of the largest

\footnotetext{
${ }^{*}$ Our memory function implementations [14], benchmarking methodology $[11,13]$ and raw measurements [10] have been open sourced.
}

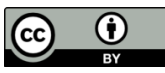

This work is licensed under a Creative Commons Attribution 4.0 International License.

ISMM '21, fune 22, 2021, Virtual, Canada

(c) 2021 Copyright held by the owner/author(s).

ACM ISBN 978-1-4503-8448-3/21/06.

https://doi.org/10.1145/3459898.3463904 computing enterprises in the world. This work increased the performance of the fleet by $1 \%$.

CCS Concepts - Software and its engineering $\rightarrow$ Software development techniques; • General and reference $\rightarrow$ Measurement; Performance; Empirical studies; Metrics.

Keywords memory functions, C standard library

ACM Reference Format:

Guillaume Chatelet, Chris Kennelly, Sam (Likun) Xi, Ondrej Sykora, Clément Courbet, Xinliang David Li, and Bruno De Backer. 2021. automemcpy: A Framework for Automatic Generation of Fundamental Memory Operations. In Proceedings of the 2021 ACM SIGPLAN International Symposium on Memory Management (ISMM '21), June 22, 2021, Virtual, Canada. ACM, New York, NY, USA, 13 pages. https://doi.org/10.1145/3459898.3463904

\section{Introduction}

Interacting with memory is an essential part of software development. At the lowest level the two fundamental memory operations are load and store, but developers frequently use higher level memory primitives such as initialization, comparison, and copy. These operations are at the base of nearly all libraries, ${ }^{1}$ language run-times,${ }^{2}$ and even programming languages constructs. ${ }^{3}$ They may be customized for some particular contexts-such as for kernel use or embedded development-but the vast majority of software depends

\footnotetext{
${ }^{1}$ e.g., std::string and std::vector in the $\mathrm{C}++$ Standard Template Library.

${ }^{2}$ Garbage collectors move memory to reduce fragmentation, reflection APIs rely on run-time type identifier comparison.

${ }^{3}$ In some $\mathrm{C}$ implementations, passing a large struct by value inserts a call to libc's memcpy.
} 
Table 1. Overview of memcpy source code language for various architectures and libc implementations.

\begin{tabular}{|c|c|c|c|c|c|c|c|c|}
\hline & نे & iो & $x^{e^{e^{c}}}$ & $\dot{\alpha}^{e^{v^{v}}}$ & $\vec{v}$ & eह & cit & $\sigma^{\nu^{5}}$ \\
\hline aarch64 & asm & asm & asm & - & - & - & - & asm \\
\hline arm & asm & asm & asm & - & - & asm & - & asm \\
\hline i386 & asm & asm & asm & asm & C & asm & - & asm \\
\hline$x 86-64$ & asm & asm & asm & asm & asm & asm & - & asm \\
\hline alpha & - & asm & - & - & - & asm & - & - \\
\hline generic & - & - & - & - & - & - & C & C \\
\hline ia64 & - & asm & - & - & asm & asm & - & - \\
\hline mips & - & asm & asm & - & - & - & - & - \\
\hline powerpc & - & asm & asm & - & C & - & - & - \\
\hline s390 & - & asm & - & - & - & asm & - & - \\
\hline sh & - & asm & - & - & asm & asm & - & - \\
\hline sparc32 & - & asm & - & asm & asm & asm & - & - \\
\hline sparc64 & - & asm & - & asm & - & asm & - & - \\
\hline
\end{tabular}

on their implementations in the C standard library: memset, memcmp and memcpy ${ }^{4}$.

In the rest of this paper, we will focus on the optimization of memcpy as the hottest of the three functions, but the same approach can be applied to the other two.

As a reminder, memcpy is defined in $\$ 7.24 .2 .1$ of the $C$ standard [21] like so: The memcpy function copies $n$ characters from the object pointed to by s2 into the object pointed to by s1. If copying takes place between objects that overlap, the behavior is undefined.

\section{Overview of Current Implementations}

\subsection{Pervasive Use of Assembly}

Memory primitives have been part of the $\mathrm{C}$ standard library for at least three decades. They have seen persistent efforts to exploit the capabilities (and work around the quirks) of each architecture as it evolved over time. Developers, who were typically well-versed in the subtleties of their target micro-architecture, would choose assembly to obtain the maximal amount of control on the resulting generated code. As a result, out of the eight main publicly available libc implementations (see Table 1), all but one use assembly to implement memcpy. As shown in Table 2, glibc ${ }^{5}$ alone has six x86-64 versions of memcpy to exploit different instruction set extensions such as SSE2, SSE3, AVX, and AVX512.

\subsection{Dynamic Loading and Relocation}

Standard C libraries are usually provided as shared libraries, which brings a number of advantages:

- it avoids code duplication-saving disk space and memory,

\footnotetext{
${ }^{4}$ memmove is not considered in this paper as: (1) it requires additional logic and (2) in our experience, its use is anecdotal compared to memcpy, memcmp and memset.

${ }^{5}$ The GNU C library.
}

Table 2. Binary size of glibc 2.31 memcpy implementations for $\mathrm{x} 86-64$.

\begin{tabular}{lr}
\hline Name & Size (bytes) \\
\hline _-memcpy_avx512_no_vzeroupper & 1855 \\
-_memcpy_avx512_unaligned_erms & 1248 \\
-_memcpy_avx_unaligned_erms & 984 \\
-_memcpy_sse2_unaligned_erms & 765 \\
-_memcpy_sse3 & 10695 \\
_-memcpy_sse3_back & 10966 \\
\hline
\end{tabular}

- it enables quick updates-this is especially important for maintenance and security reasons,

- to the developer, it accelerates the compilation process by reducing link time.

Shared libraries also come with costs:

- Symbols from shared libraries can't be resolved at compile time and they need extra run-time infrastructure. Modern Linux systems and x86-64 in particular implement shared libraries using Position Independent Code (PIC) [5-7]. PIC avoids costly relocation of function addresses at load time by using an extra indirect call through the Procedure Linkage Table (PLT). This indirect call hurts performance and increases instruction Translation Lookaside Buffer (iTLB) misses.

- Functions imported from shared libraries are not visible to the linker. This prevents optimizations such as inlining and Feedback-Directed Optimization (FDO). We discuss FDO in more detail in Section 2.4.

In Google data centers, applications are statically linked and their size is typically in the hundreds of megabytes. Statically linking the memory primitives only marginally increases the binary size and overcomes the aforementioned problems.

\subsection{Run-Time Dispatch}

As CPUs and Instruction Set Architectures (ISAs) evolve, new instructions become available and performance of older instructions may improve or degrade. Unless a libc is built for a specific CPU model, it must accommodate the older models but it should also provide an optimized versions for newer CPU models. To this end, Linux libraries use a run-time dispatching technique on top of PLT called IFUNC [26] that is commonly used by glibc. On the first call to an IFUNC, the indirect address-stored in the Global Offset Table (GOT)points to a custom resolver function that determines the best implementation for the host machine. The resolver then overrides its own GOT entry so that subsequent calls access the selected function. After the setup phase, an IFUNC has the same indirect call costs and limitations as a function from a shared library, even if it is linked statically. 


\subsection{Feedback-Directed Optimization}

The most significant optimizations in recent years came from the mainstream adoption of Feedback-Directed Optimization $^{6}$ (FDO) with global efficiency improvements typically over $10 \%$ [15]. FDO relies on a measured execution profile to make informed compilation decisions ${ }^{7}$ based on how the application actually executes the code. Because memory primitives are so pervasive among all applications, they are especially well covered by sampling profilers ${ }^{8}$ and could greatly benefit from FDO.

However, FDO can only be applied to the parts of the application for which source code is available to the compiler/linker. In particular, it can't be applied to dynamically linked functions and IFUNCs. Moreover, FDO does not understand and is not allowed to modify code written in assembly. This effectively means that the way memory functions are implemented on most systems prevents them from benefiting from one of the most efficient optimization techniques.

\section{Our Approach}

Based on our analysis of the shortcomings of current implementations, we design our implementation primitives around two main principles:

- High-level description. The implementation should be written using a high-level language-we chose $\mathrm{C}++$ for its expressiveness. This makes the code shorter and more readable, and it allows the compiler to use advanced optimization techniques, including FDO. When needed, we examine the generated code to debug performance issues, but never write assembly directly.

- Data-based optimization. On top of fleet-wide execution profiles, we record run-time values of the memcpy size argument for several representative workloads. We use the measured size distribution to automatically select the best set of elementary strategies and design the decision logic driving the function.

\subsection{Measuring size Distributions}

There are several ways of collecting the run-time values for the size argument:

1. Manually instrument our memcpy implementations.

2. Rely on compiler's instrumentation and value profiler.

3. Rely on profiling infrastructure.

Solutions 1 and 2 require a special (instrumented) build of each application and add an extra run-time cost which is impractical for use in production. Instead, we make use of existing profiling infrastructure: the Linux perf tool [27]. This is an interface to the Linux performance monitoring

\footnotetext{
${ }^{6}$ In some contexts, FDO is also called Profile-Guided Optimization.

${ }^{7}$ Examples of what can be achieved are: determining which functions to inline, or how to lay out the code to improve locality and reduce branching. ${ }^{8}$ Modern datacenters continuously run sampling profilers on their workloads as a part of standard operations [22].
}

subsystem that can attach to a running process and collect profiling data without a need to recompile or restart the application. Among other features, it allows sampling register values at certain points during the execution of the program. In our case, we set it up to sample the RDX register right after a call instruction. Under the System V x86-64 ABI [25], this register contains the size argument of memcpy. Since memcpy is statically linked, its address in the binary is known at compile time, so we can easily filter samples belonging to memcpy and gather them into a histogram.

\subsection{Performance Definition}

There are many dimensions to consider when designing a memory function implementation. Contrary to existing implementations that usually optimize for throughput, ${ }^{9}$ we will focus on latency and code size as they are most important for our use cases. Code size is measured with the help of the nm linux command and latency is measured through a custom benchmarking framework. We define latency as the running time of the memory operation for a given hardware in a given context.

\subsection{Benchmarking}

Measuring the performance of memory functions is both hard and critical, so we devote a whole section to our benchmarking methodology. In the context of Google, we can rely on fleet-wide profiling to measure the efficiency of an implementation. However, linking statically means that we have to wait for all applications to be released before seeing the results, which is not practical.

The aim here is to provide an accurate and reproducible measurement of latency. To do so we heed the following design principles:

1. Measuring instrument. We make use of the Time Stamp Counter (TSC). It is the most precise clock available: a hardware register located on the CPU itself, counting the number of CPU cycles elapsed since boot. There are two versions of the TSC on x86-64 processors. One is in-core and ticks at a rate depending on a number of factors (power saving mode, defined maximum and minimum frequency, thermal regulation); the other one is Uncore ${ }^{10}$ which ticks at a fixed frequency usually referred to as ref cycles. Most high end CPU architectures provide similar functionality (SPARC, ARM, IBM POWER). The Uncore counter's frequency is about the same as that of the processor in non-turbo mode. We use the Uncore counter to account for uniformly elapsing time.

2. Frequency Scaling. The Operating System usually provides a way for the user to control the CPU core frequencies (i.e., Scaling Governors under Linux), but

\footnotetext{
${ }^{9}$ Number of bytes processed per unit of time.

${ }^{10} \mathrm{https}: / /$ en.wikipedia.org/wiki/Uncore
} 
the CPU may reduce its frequency automatically under some workloads $[19,24]$. By instructing the CPU to run at the maximum allowed frequency and using the uncore TSC, we make sure to account for potential frequency reductions.

3. Operating System. To lower the impact of interruption and process migration, we pin the benchmarking application to a reserved core. ${ }^{11}$

4. Compiler perturbations. Clever dead-code and dependency analyses performed automatically by the compiler may cancel or distort the measurement. To this end we use techniques such as the escape inline assembly described by Carruth [8].

5. Memory Subsystem. On modern non-embedded systems, RAM bus frequency is only a fraction of the CPU core frequency. This well-known bottleneck is balanced out by several levels of caching. The number, size, and speed of each of these layers makes it hard to attribute the result of the measurement to the algorithm itself. For this reason, our framework takes extra care to fit all the needed data into the L1 cache. The results are slightly idealized but more reproducible and comparable between CPUs of different cache sizes.

6. CPU. At the CPU level, out-of-order execution, superscalar architecture, Macro-Operation Fusion (MOP Fusion), Micro-Operation Fusion, and speculative execution make it harder to correlate TSC with individual instructions. To mitigate these imprecisions and increase the Signal to Noise Ratio (SNR) we run enough iterations of each function to get noise down to $\pm 1 \%$. Note that the repeated execution will bias the results as the CPU will learn the branching pattern perfectly. Consequently, we randomize the size of the operation as well as the source and destination pointers. Randomization is pre-computed and stored in an array so it is not accounted for in the measurement. The size of the array is large enough to prevent quantization effects but small enough to keep all data in L1.

Additionally, it is worth noting that a number of effects do play a role in production and are not directly measurable through microbenchmarks. We end this section with the following known limitations:

1. The code size of the benchmark is smaller than the hot code of real applications and it doesn't exhibit instruction cache and iTLB pressure as much.

2. For the reasons stated in item 5 above, the current version of the benchmark does not handle large operations spanning multiple cache levels.

\footnotetext{
${ }^{11}$ If the processor supports Simultaneous MultiThreading (SMT) all the logical CPUs sharing the same core should be reserved as well, or SMT should be disabled altogether.
}

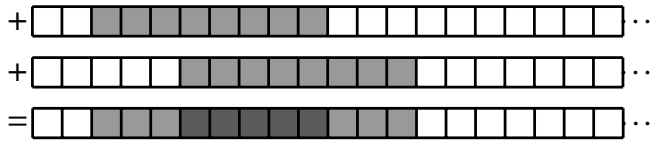

Figure 1. Example Overlapping operation on 11 bytes. Bytes from the source location are copied using two 8-byte block operations that partially overlap. The five bytes in the middle are copied twice.

\subsection{Generic Access Strategies}

At a high level, a memcpy implementation is assembled from a set of simpler copying strategies (elementary strategies) along with its associated decision logic that selects the best copying strategy for a given size.

By analyzing the code of available implementations and through experimentation, we identified the following patterns for processing memory. In this section, we list these strategies and describe their specifics.

\subsubsection{Block Operation}

A Block Operation consists of accessing a fixed amount of memory of size BlockSize. To be efficient, BlockSize should correspond to sizes natively supported by load and store instructions of the CPU architecture. It is the simplest access pattern and is implemented using the simple load and store instructions provided by the ISA.

\subsubsection{Overlap Operation}

This access pattern is the composition of two Block Operations that may partially overlap, and it is a central component of efficient implementations. This approach has several advantages. First, a single access pattern involving two operations of size $N$ can handle a range of sizes $\in[N ; 2 \times N]$. Second, modern processors offer memory addressing modes that render this pattern efficient in practice.

Figure 1 shows an example of Overlap Operation for 11 bytes. As depicted by the darker cells, one or more bytes may be accessed several times. While this strategy is valid for implementing memcpy, such a technique is not suitable when volatile ${ }^{12}$ semantics apply.

\subsubsection{Loop Operation}

The Loop Operation involves a repetition of Block Operations followed by a trailing Overlap Operation. Note that up to BlockSize - 1 bytes may be accessed twice. Figure 2 shows an example of a Loop Operation.

\subsubsection{Aligned Loop Operation}

The Aligned Loop Operation starts with a Block Operation to ensure that reads are aligned. It is then followed by a

\footnotetext{
${ }^{12}$ Note that the volatile keyword in $\mathrm{C}$ presents a number of issues and is considered for deprecation [4].
} 


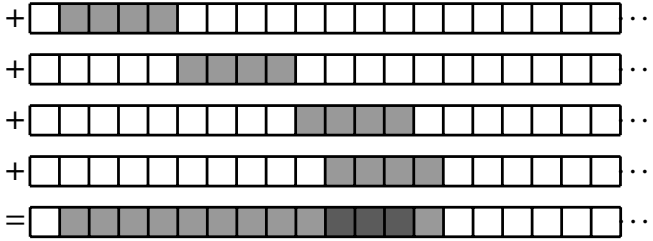

Figure 2. Example Loop Operation on 13 bytes. The first 12 bytes are copied using three non-overlapping 4-byte Block Operations. The remaining one byte is copied using a partially overlapping 4-byte Block Operation. Three bytes are accessed twice.

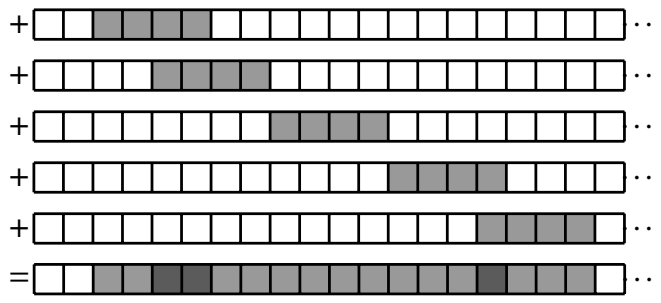

Figure 3. Example Aligned Loop Operation on 17 bytes. Two partially overlapping operations are used the beginning and the end. All remaining bytes are handled using aligned non-overlapping Block Operations.

Loop Operation. Note that in a worst case scenario, up to BlockSize -2 bytes may be accessed three times. Figure 3 shows an example of an Aligned Operation.

\subsubsection{Special Instructions}

Some ISAs provide primitives to accelerate string manipulation. For example, x86-64 offers specialized instructions for copy (rep mov), initialization (rep sto) and comparison (repe cmps). The performance of these instructions varies greatly from microarchitecture to microarchitecture [28] but it is important to consider them as their footprint is so small they could be easily inlined everywhere. In the rest of this paper we refer to these special instructions as accelerators.

\subsection{Run-Time Size Coverage}

The Generic Access Strategies we presented in the previous section can handle one or more sizes, Table 3 lists their runtime size coverage in terms of BlockSize.

These strategies can be composed in different ways to build a full implementation covering the whole size_t range [0,SIZE_MAX]. By way of illustration, a possible coverage is given in Table 4.

To find the best memcpy implementation, we use a constraint solver to enumerate all valid memcpy implementations matching our specification. We consider only memory functions that subdivide the range [0,SIZE_MAX] into smaller
Table 3. Size coverage for a given strategy

\begin{tabular}{lr}
\hline Operation & Size range \\
\hline Block & [BlockSize, BlockSize $]$ \\
Overlap & {$[$ BlockSize, $2 \times$ BlockSize $]$} \\
Loop & {$[$ BlockSize,$+\infty]$} \\
Aligned Loop & {$[$ BlockSize,$+\infty]$} \\
Accelerator & {$[0,+\infty]$} \\
\hline
\end{tabular}

Table 4. An example of coverage for a memory operation

\begin{tabular}{lrr}
\hline Size range & Operation & BlockSize \\
\hline$[0,0]$ & - & - \\
{$[1,1]$} & Block & 1 \\
{$[2,4]$} & Overlap & 2 \\
{$[5$, SIZE_MAX $]$} & Loop & 4 \\
\hline
\end{tabular}

regions and cover each of them using one of the strategies described in Section 3.4 using the following schema:

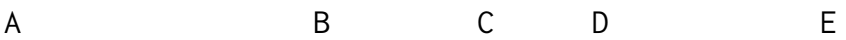
| individual sizes | overlap | loop | accelerator | where $A, B, C, D$ and $E$ are bounds separating the regions.

If the run-time copy size is in [A, B) the copy will be handled with the individual sizes strategy, in $[B, C)$ with the overlap strategy, and so on. A and E are anchored to 0 and SIZE_MAX respectively to make sure the whole range is covered (eq. 1), and we make sure that the bounds are ordered (eq. 2).

$$
(A=0) \wedge(E=\text { SIZE_MAX })
$$

$$
(A \leq B) \wedge(B \leq C) \wedge(C \leq D) \wedge(D \leq E)
$$

When the two bounds of a region are equal, e.g., when $A=B$, the region is empty and the corresponding strategy is not used in the generated function.

The individual sizes region handles each run-time size individually with a Block Operation. In this study we restrict $B$ to small values as larger values substantially increase code size.

$$
(A=B) \vee(B \leq 8) \vee(B=16)
$$

The overlap region handles run-time sizes from $B$ to $C$ by using one or more Overlap Operations. In this study we explore all overlap regions for size $\in[4 ; 256]$ as well as the empty region.

$$
(B=C) \vee\left\{\begin{array}{l}
B<C \\
B \in\{4,8,16,32,64,128\} \\
C \in\{8,16,32,64,128,256\}
\end{array}\right.
$$

The loop region employs the aligned loop strategy with a run-time BlockSize $\in\{8,16,32,64\}$. If $C=D$, we also force loop_block $=0$ to remove redundant implementations. 


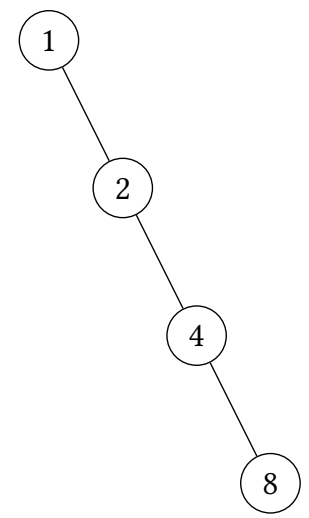

(a) Simple tree processing small sizes first

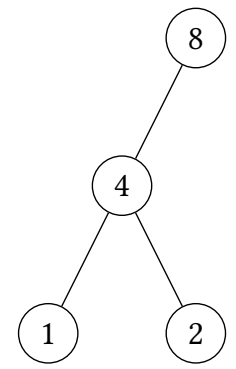

(b) An optimized tree where most values are above 8
Figure 4. A depiction of two branching strategies

$$
\left.\begin{array}{r}
\text { loop_block }=0 \\
C=D
\end{array}\right\} \vee\left\{\begin{array}{l}
\text { loop_block } \in\{8,16,32,64\} \\
C>2 \times \text { loop_block } \\
D-C>\text { loop_block }
\end{array}\right.
$$

The accelerator region makes use of special instructions where available. We allow accelerator to start for small sizes or amongst a set of discrete values.

$$
(D \leq 16) \vee(D \in\{32,64,128,256,512,1024\}) \vee(D=E)
$$

\subsection{Branching Pattern}

Once a coverage has been determined, it is necessary to decide which branch should be favored to lower the whole operation latency. A simple approach is to make sure that small sizes are processed first so that the cost of branching is proportional to the amount of bytes to process (Figure 4a). Another possibility is to prioritize sizes that occur the most. To this end, we make use of Knuth's Optimum Binary Search Trees [23] that constructs a binary search tree producing code with the shortest expected control height for a given set of probabilities (Figure 4b).

The implementation can also explore a more elaborate model to represent the cost of compares, branch instructions, and taken branches. A hybrid expansion approach can also be used. For instance when a sequence of individual sizes gets dispatched to Block Operations, all sizes can be lumped together and share one jump table. Note that this approach will be the most effective when it is used for inlining-using per-callsite profile data.

\subsection{Optimized Memory Functions}

The usage of memory primitives varies widely depending on the use case. One extreme case is the Linux kernel that spends a very significant proportion of its time clearing or copying memory pages, which are always 4096 bytes in length. Just like $l i b c$, the Linux kernel provides several implementations, with a dynamic selection mechanism. In contrast, for typical applications running on Google fleet, we've found that sizes are very biased towards zero (see Section 4.1). The best algorithm for the Linux kernel is not necessarily optimal for other applications. In this section we provide a principled approach to design efficient memory functions tailored to a given environment:

1. We first model the function in terms of its coverage strategies as described in Section 3.5, and use the Z3 Solver [16] to enumerate all valid coverages. Each of them is described in terms of its parameters A, B, C, D, $E$, and loop_block, and whether we use an optimized branching pattern or not (Section 3.6).

2. We generate the source code for all of them and encode the values of the parameters into the function name-this allows us to generate all functions in a single file and quickly identify and understand the implementation.

3. The code is then compiled and benchmarked on all relevant microarchitectures using synthetic distributions observed in production.

4. The best performing implementation is chosen, in the case of a fleet of heterogeneous microarchitectures we pick the implementation that minimizes the overall latency.

Not only does this approach allow the functions to be optimized for a particular application (or set of applications) but it also adapts to special requirements like special compilation flags, new microarchitectures or new CPUs. The model described here generates 290 different functions which are benchmarked in one or two hours. It is straightforward to extend the exploration of the implementation space.

The techniques mentioned here are related to the domain of auto-tuning which has been an important field of research in High Performance Computing (HPC) [3] and Signal Processing libraries $[17,18,30]$. To our knowledge, the only related work in this domain is the one from Ying et al. [31], focusing on optimal generation of assembly for Reduced Instruction Set Computer (RISC).

\section{Results}

\subsection{Memfunctions Size Distributions}

The instrumentation procedure described in Section 3.1 introduces a small overhead (1-3\%) on the application itself during the time that the profiler is active, due to the overhead introduced by perf_events profiling. A 30-second profiling session typically gathers enough data for analysis, depending on the frequency with which the application calls the memory operations. Figures 5, 6, and 7 shows the cumulative probability distribution for sizes of the biggest users of memcpy, memcmp and memset at Google. The data is publicly available [12]. Table 5 summarizes these figures and shows 


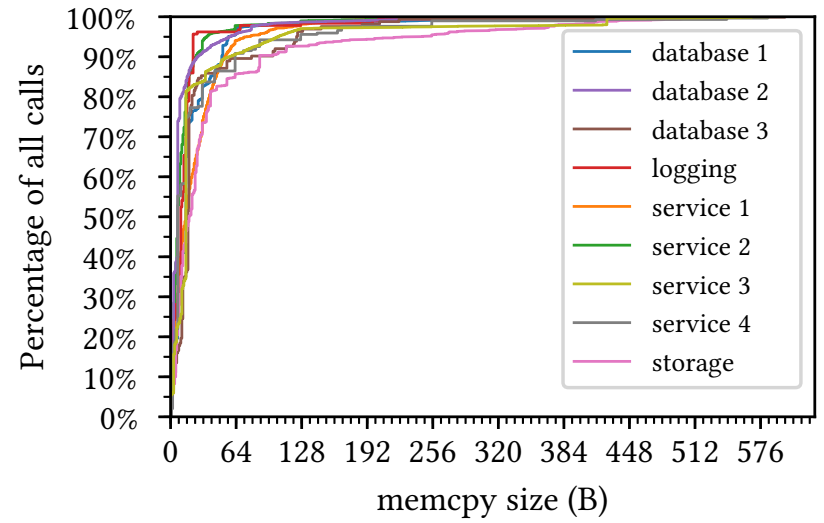

Figure 5. Cumulative histogram of memcpy size for various Google workloads.

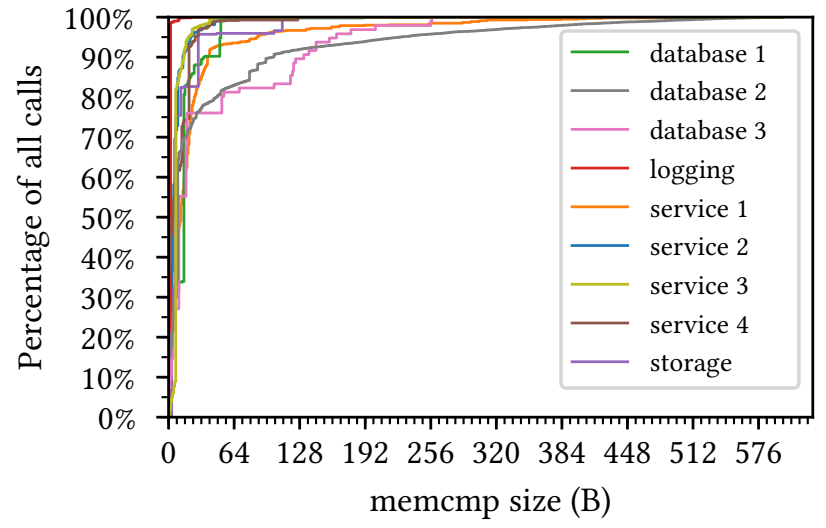

Figure 6. Cumulative histogram of memcmp size for various Google workloads.

Table 5. Percent of calls below certain size values

\begin{tabular}{lrr}
\hline Function & $\%$ of calls $\leq 128$ & \% of calls $\leq 1024$ \\
\hline memcpy & $96 \%$ & $99 \%$ \\
memcmp & $99.5 \%$ & $100 \%$ \\
memset & $91 \%$ & $99.9 \%$ \\
\hline
\end{tabular}

that the majority of calls to memory functions act on a small number of bytes.

The fact that the size distribution is strongly skewed towards small sizes advocates optimizing for latency instead of throughput. We have seen previously that relocation and run-time dispatch introduce an indirect call which delays processing. For example, a trivial zero size memory operation would still have to go through address resolution and perform the indirect call before returning to the calling code. To remove this latency entirely we link the memory primitives

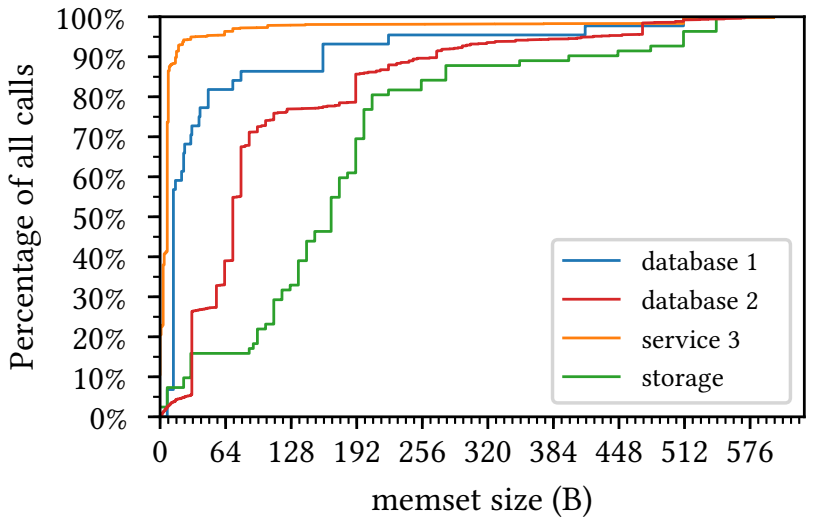

Figure 7. Cumulative histogram of memset size for various Google workloads.

statically inside the binary and refrain from using IFUNC. This comes with the disadvantage that we can no longer pick the best implementation for the CPU at run-time; this choice has to be made during compilation. Arguably, for distributions skewed towards small sizes, the use of wider load and store instructions available on newer microarchitectures doesn't matter as much. In situations where it is important, this can be mitigated by providing several binaries covering the diversity of the fleet.

\subsection{C++ Library}

In Listing 1, we provide Block, Overlap and Aligned Loop C++ implementations of the copy operation. The individual building blocks are unit tested for correctness, buffer overflow and maximum number of accesses per byte. Note that the use of _-builtin_memcpy_inline is a compiler intrinsic function that is semantically equivalent to a for loop copying bytes from source to destination-i.e., has the semantic of memcpy. Its use is necessary to prevent the compiler from recognizing the memcpy pattern which leads to a reentrancy problem [9].

\subsection{Fleet-Wide Manual Implementation}

Now that we have defined the primitive $\mathrm{C}++$ copy functions, we can assemble them to handle all run-time sizes. Listing 2 shows a generic yet efficient implementation of memcpy that has been tuned by trying out different building block combinations and parameters. Full source code is available in [14]. Note that this version handles small sizes first. In Section 4.4, we will take a closer look at size distributions and explore optimized branching patterns.

A note on the use of AVX instructions. Although a large portion of Google fleet supports the AVX instruction set extension, their use is restricted to prevent important slowdown due to frequency reduction $[19,24]$. To that end 


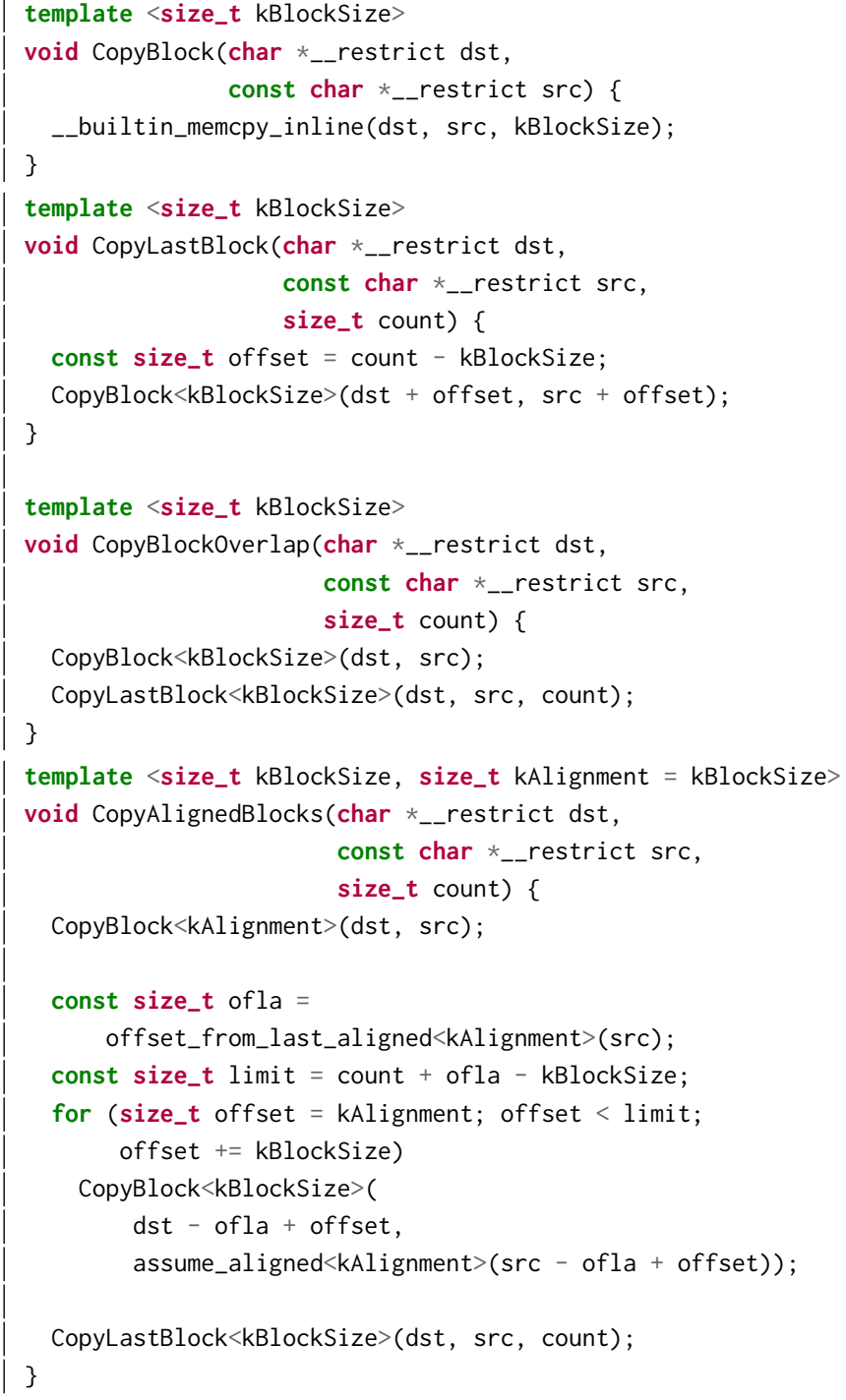

Listing 1. Real C++ code for various copy operation strategies

we make use of LLVM's -mprefer-vector-width=128 option that limits the width of generated vector instructions. For this reason, the x86-64 generated code (clang 12, options -03 -mcpu=haswell -mprefer-vector-width=128) will not use AVX. It is still quite compact (377 bytes) and fits in six cache lines.

Performance. It is important to point out that although this implementation does not use all the techniques applied by glibc (e.g., non temporal move or rep mov) [29], it performs better on Google workloads. As suggested in [2], the smaller memory footprint compared to glibc (377B vs 765B, see Table 2) allows more application code to stay in the instruction cache. Indeed memcpy, memcmp, and memset are called on a regular basis and can contribute to instruction cache pressure, evicting previous application code from L1.

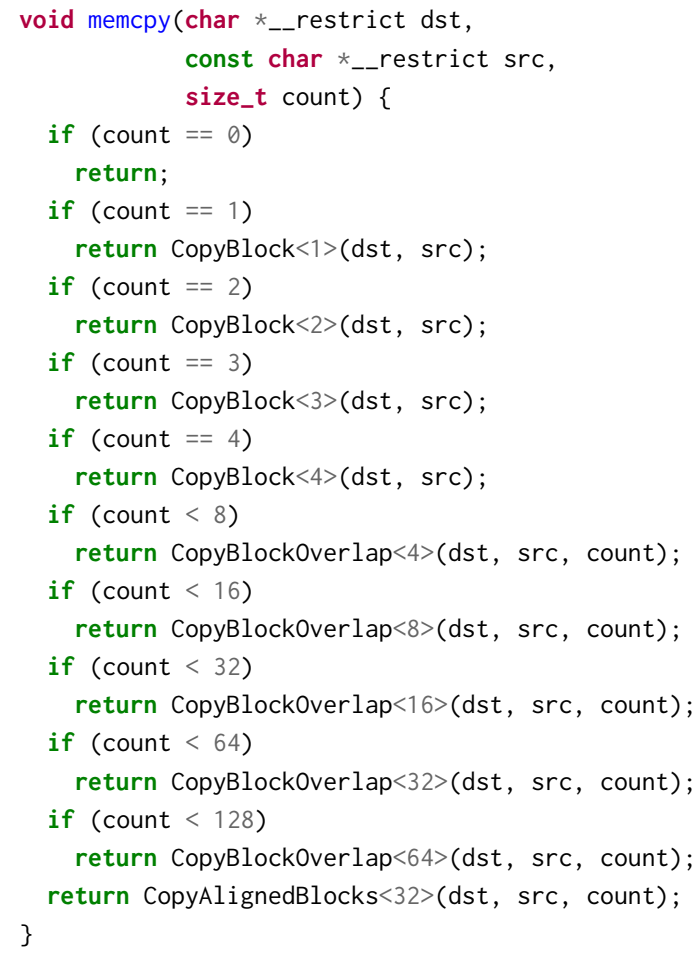

Listing 2. an efficient yet readable implementation of memcpy

Using this version of memcpy and associated memcmp and memset improved the throughput of one of our main services by $+0.65 \% \pm 0.1 \%$ Requests Per Second (RPS) compared to the default shared glibc. Overall we estimate that this work improves the performance of the fleet by $1 \%$.

\subsection{Fleet-Wide Autogenerated Implementations}

As illustrated in Figure 5, most of the sizes for memcpy at Google are heavily skewed toward small values. The most occurring ones being spread between 0 and 32 with spikes depending on specific workloads. For the optimized branching pattern (Section 3.6) we use the sum of the measured size probability distribution; giving an equal weight to each of them. The code is compiled and benchmarked on a dedicated pool of machines with isolated cores running the performance frequency governor. We benchmark the following x86-64 microarchitectures: Intel Sandybridge, Intel Ivybridge, Intel Broadwell, Intel Haswell, Intel Skylake Server (SKX) and AMD Rome. The benchmarks make use of the rep mov instruction and a total of 290 memcpy configurations. We measure the average latency for each of the 9 size distributions of Figure 5 and an additional synthetic distribution for larger sizes (uniformly distributed sizes between 384 and 4096). We produce 1000 samples for each of them to get a sense of the statistical distribution. We show a sample of the distributions in Figure 8. 


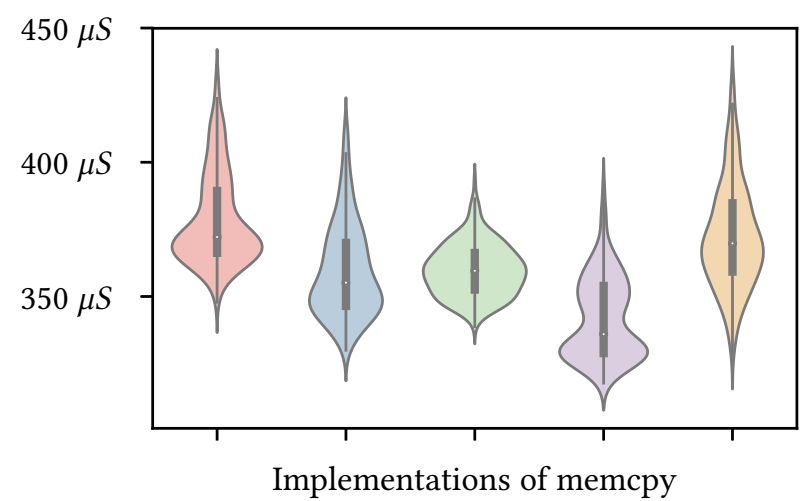

Figure 8. Distributions of latency measurement for several memcpy implementations as measured on SKX for service 1.

In the context of this paper, we reduce the thousand samples to a single median value ${ }^{13}$ as this provides a total order over the set of functions. For each distribution we derive a speedup ratio by dividing each median latency by the one of the $g l i b c^{14}$-which is our baseline implementation. We use the geometric mean (geomean) of these speedups to represent the performance of a particular implementation over the baseline and across the selected distributions. In the rest of the paper, we refer to this metric as the score of the function.

This systematic exploration exhibits implementations that perform about $+10 \%$ faster than our manual implementation for four out of six microarchitectures in our particular environment (combination of workloads and special compilation options). For Haswell and Broadwell, the manual implementation is already close to the optimum. In Figure 9, we plot the score for the 290 autogenerated x86-64 memcpy implementations as measured on Skylake Server.

Key Takeaways. Overall, the best performing functions for x86-64 rely on a small individual sizes region $(B=4$ or 8 ) followed by a set of overlapping copies up to 256 bytes while an aligned copy loop using blocks of 64 bytes helps accommodate the bigger sizes. The rep mov instruction may be useful for even bigger copies depending on the microarchitecture but it's usually on par with implementation relying exclusively on aligned copy loop. It is to be noted that the worst performing functions for our workloads are the ones that rely almost exclusively on the specialized rep mov instruction.

Timings. The whole process takes a few hours. The enumeration and code generation for all valid implementations takes a few seconds. The duration of the benchmarking process ranges from tens of minutes to a few hours, depending on a number of parameters such as: the number of samples,

\footnotetext{
${ }^{13}$ Considering that some distributions of latency measurement are multimodal we acknowledge that a better metric is desirable.

${ }^{14} \mathrm{GNU}$ C Library 2.19 with local modifications.
}

Table 6. Score for worst, manual implementation and best memcpy functions taking the system glibc as a baseline.

\begin{tabular}{lrrr}
\hline & worst & manual & best \\
\hline Rome & 0.42 & 1.35 & 1.43 \\
Skylake Server & 0.54 & 1.38 & 1.53 \\
Haswell & 0.52 & 1.39 & 1.40 \\
Broadwell & 0.52 & 1.42 & 1.43 \\
Ivybridge & 0.57 & 1.22 & 1.34 \\
Sandybridge & 0.26 & 1.23 & 1.35 \\
Neoverse-N1 & 0.82 & 1.21 & 1.36 \\
\hline
\end{tabular}

the number and shapes of the distributions, the number and efficiency of the generated functions, and the number and the performance of the considered microarchitectures. In this study, we used 1000 samples, 10 distributions, 290 functions and 6 microarchitectures. The single threaded benchmark is launched on the 6 microarchitectures in parallel and the results are available in under two hours.

Open Source. One of our goals is to make our improvements available publicly to people who do not have access to Google fleet. The community should be able to measure performance and contribute improvements, so we released the methodology and the source code of our benchmark as open source $[11,13]$.

Results. The worst, manual implementation and best performing implementations are summarized in Table 6. It is worth mentioning that the optimized branching pattern does not yield a systematic performance improvement and seems to be quite dependent on the processor microarchitecture. Further investigation is required to determine the usefulness of this optimization.

Case Study. To test the robustness of our method on a different architecture, we conducted the same experiment on an ARM Neoverse-N1 machine. The ARM ISA has no instructions similar to rep mov, so the number of generated functions drops to 60 . We complement this set of functions with a particular implementation from ARM Optimized Routines [1] to check how close we get to optimized assemblythis implementation is statically linked. In our context, our top performing function has an overall speedup of 1.36 over the system's glibc ${ }^{15}$ which is about $+8 \%$ faster than the handwritten assembly scoring 1.26 . We note that each of them has different strengths and weaknesses depending on a particular size distribution.

\subsection{Tailored Implementations}

In the two previous sections, we have shown how we can write a generic implementation of memcpy that works efficiently across the fleet. However, we do not claim that all workloads are similar to those that we run at Google. In this

\footnotetext{
${ }^{15} \mathrm{GNU}$ C Library 2.27 with local modifications.
} 


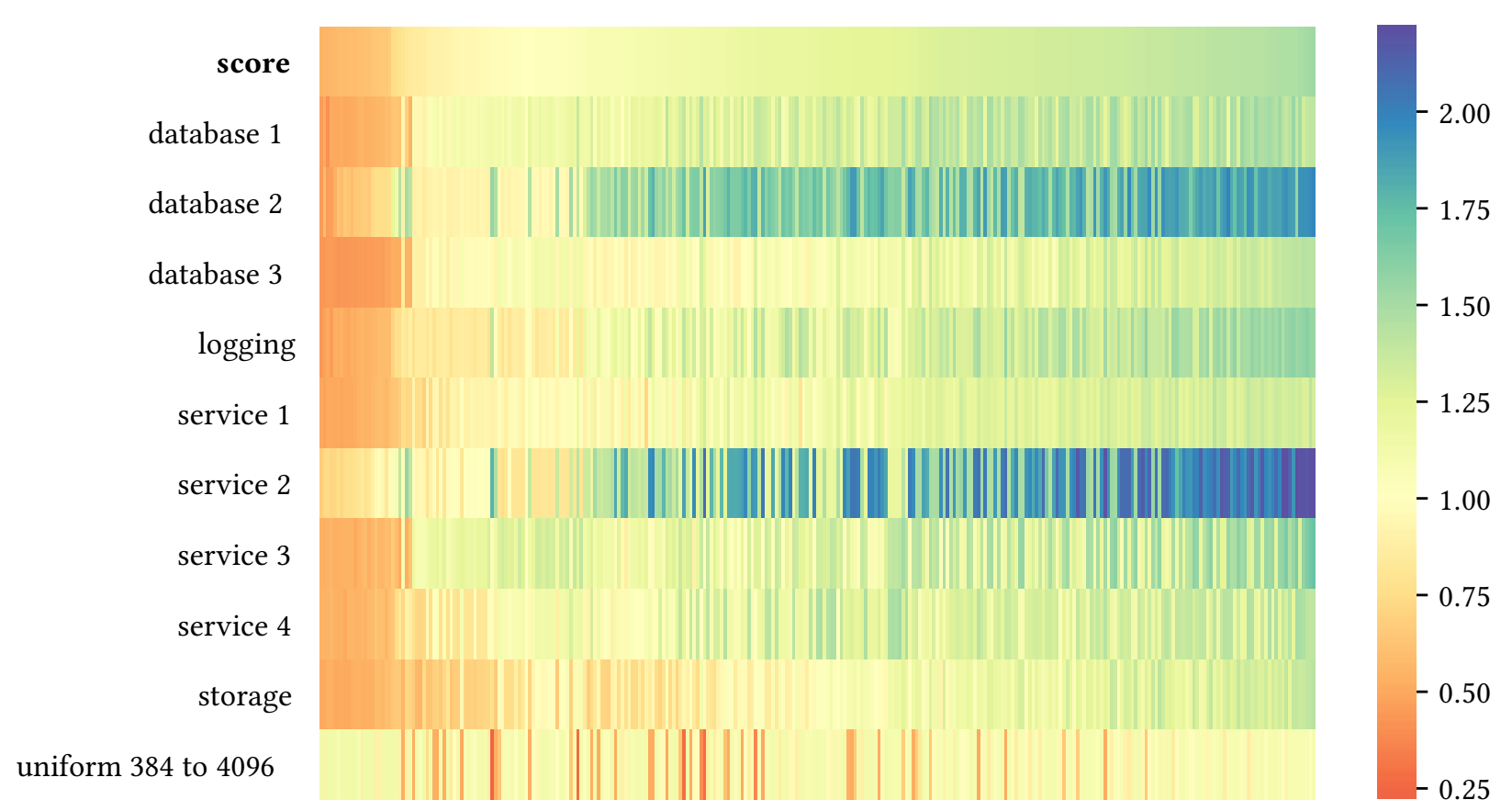

Figure 9. The speedups of the 290 generated memcpy functions for individual applications on SKX over glibc (score $\in$ $[0.53,1.53])$.

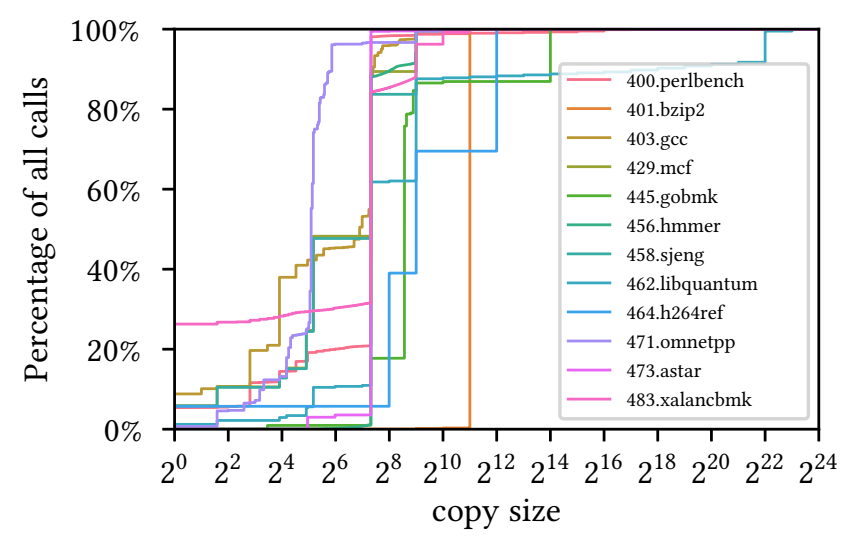

Figure 10. Cumulative distribution of memcpy sizes for each SPEC int benchmark (ref set). Note the log scale.

section, we show how our approach can be used to derive efficient implementations for a specific workload.

The SPEC benchmark suite [20] is a widely used set of benchmarks that covers a diverse set of compute-intensive workloads from real-world applications. Figure 10 shows the distribution of memcpy sizes for each benchmark of the ref data set.

One important thing to notice is that even though most workloads make small copies, some of them (e.g., h264ref, libquantum) perform a significant number of large copies.
Table 7. Percentage of the time spent in memcpy for each SPEC int benchmark (ref dataset) using the glibc implementation on Haswell

\begin{tabular}{lr}
\hline benchmark & \% time in memcpy \\
\hline 400.perlbench & $1.1 \%$ \\
401.bzip2 & $0.2 \%$ \\
403.gcc & $0.3 \%$ \\
429.mcf & $0.0 \%$ \\
445.gobmk & $0.3 \%$ \\
456.hmmer & $0.0 \%$ \\
458.sjeng & $0.0 \%$ \\
462.libquantum & $0.0 \%$ \\
464.h264ref & $24.3 \%$ \\
471.omnetpp & $0.0 \%$ \\
473.astar & $0.0 \%$ \\
483.xalancbmk & $3.3 \%$ \\
\hline
\end{tabular}

Additionally, Table 7 shows that for some of them, memcpy represents a very significant portion of the total time for the benchmark. Among all these examples, 464.h264ref is an outlier as it has both a non-standard distribution of sizes, and spends a large amount of time in memcpy (about $24 \%$ of total cycles).

We focus on 464. h264ref to demonstrate the power of this techniques on an application with very specific needs. 


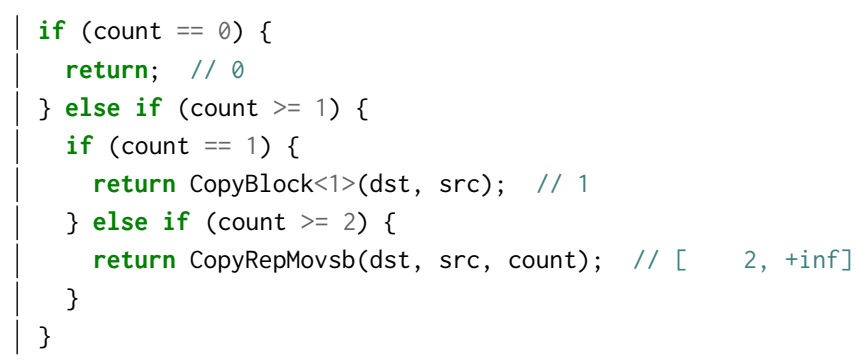

Listing 3. The auto-generated implementation selected by automemcpy for 464.h264ref.

Table 8. Performance of the glibc, fleet-wide and specialized memcpy for the 464.h264ref SPEC benchmark, on HSW. Intervals are given with $95 \%$ confidence, and the speedup is defined as the ratio between the time for glibc and that for the implementation (larger is better)

\begin{tabular}{lccc}
\hline $\begin{array}{l}\text { memcpy } \\
\text { implementation }\end{array}$ & $\begin{array}{c}\text { \% time in } \\
\text { memcpy }\end{array}$ & $\begin{array}{c}\text { memcpy } \\
\text { speedup }\end{array}$ & $\begin{array}{c}\text { total } \\
\text { speedup }\end{array}$ \\
\hline glibc & $24.35 \% \pm 0.13$ & 1.00 & 1.00 \\
fleet-wide & $25.14 \% \pm 0.10$ & 0.96 & 0.99 \\
specialized & $15.05 \% \pm 0.15$ & 1.80 & 1.11 \\
\hline
\end{tabular}

To generate an optimized memcpy implementation, we collected a size profile when running the benchmark, and ran automemcpy on the result. The framework selected the implementation shown in Listing 3. Intuitively the framework detects, based on data, that memcpy sizes are either 0,1 , or very large, and so it optimizes for these two cases. The resulting code is extremely simple and small.

We ran the 464.h264ref benchmark with the original glibc implementation, our fleet-wide implementation, and the specialized implementation generated by automemcpy. All experiments were performed on a 6-core Xeon E5 running at $3.50 \mathrm{GHz}$ with $16 \mathrm{MB} \mathrm{L} 3$ cache. The results are summarized in Table 8. Unsurprisingly, our fleet-wide implementation is worse than the original glibc, because the implementation optimizes for a different size distribution than that of 464. h264ref. However, the specialized implementation outperforms the one from glibc by a factor of 1.8 , resulting in the benchmark's running more than $10 \%$ faster overall.

Keep in mind that the auto-generated memcpy implementation is only going to be efficient as long as the size distribution of the data does not significantly differ from the one on which is was trained. Therefore, such specializations must be regenerated periodically. Ideally, they should be part of the release process of the target application.

\section{Limitations and Further Work}

\subsection{Collected Data}

Since the resulting implementation depends on the collected data, it is critical that the measured size distribution be as representative as possible of the real run-time behavior. In the context of this paper, we used nine archetypal workloads and-as we have seen in Section 4.1-these distributions are all very similar for memcpy. However, it is possible that other workloads use a different size distribution. We plan on working on a more systematic approach to collect size distribution data for all workloads-weighted by their prevalence in the fleet.

\subsection{Heterogeneous size Distributions}

As seen in Section 4.5, building tailored implementations for a binary can greatly improve its global performance. Some particular workloads may use an entirely different size distribution, some may also have a different size distribution per call site. We want to explore letting the compiler automatically generate the proper implementation by itself, based on sampled run-time data. One can even envision generating multiple specialized implementations for one application, based on the call site context. They could be inlined or shared across multiple contexts via cloning.

\subsection{Limitations of Benchmarking}

Overall, we pick the function that maximizes the score, but a number of effects do play a role in production that are not directly measurable through benchmarks. For instance, the code size of the benchmark is smaller than the hot code of real applications and doesn't exhibit instruction cache pressure as much. When a candidate function is selected, it is crucial to also test it in production and verify that the performance gains are realized.

\subsection{Framework Extensions}

The framework presented in this paper can be extended in a number of ways:

1. By design, the benchmarking methodology imposes a restriction on the number of bytes to process: all data movement should stay within L1 to prevent accounting for the specifics of the memory subsystem. If bigger sizes are needed, different benchmarking methods must be used as we can no longer rely on repeating the measurement to increase SNR.

2. More Generic Access Strategies (Section 3.4) can be added if necessary. One can think of new processor capabilities like SVE for recent ARM processors or special instructions hinting the memory subsystem like PREFETCHTO or PREFETCHNTA.

3. The Constraint System developed in Section 3.5 can be adapted to explore other parts of the implementation space (e.g., use more discrete values, use of unaligned 
loops, use of aligned loops with different values for BlockSize and Alignment, alignment on destination rather than source for aligned loop).

4. If the number of possible implementations becomes so great that a systematic exploration is no more practical, the problem can be viewed as an online optimization problem.

\section{Conclusion}

In this paper, we have devised a method to quickly iterate and explore the design space of memory primitives. It relies on the composition of elementary strategies activated by a decision logic written in $\mathrm{C}++$. This is a departure from the "hand-crafted assembly" approach where decision logic, elementary strategies and instruction selection are all intertwined. We have demonstrated that our implementation matches the performance of hand-written assembly and ultimately provides better performance, because it is much easier to optimize and tune for a given workload. The high level implementation is also easier to test and maintain, as logical units can be tested separately.

Furthermore, we present a method to automatically generate a set of valid $\mathrm{C}++$ implementations of memcpy and evaluate them through a specific benchmark reproducing the size distributions measured in production. It allows finding and deploying the functions that perform best in a particular environment. This process requires minimal human effort, which makes it easy to repeat it whenever the usage patterns of the functions change. This method is suitable for optimizing both fleet-wide deployment (Section 4.4) and particular applications (Section 4.5).

Finally, we have demonstrated that we can improve the performance of applications by taking into account the specific run-time size distributions of their memory primitives. In specific benchmarks (SPEC 464.h264ref) we were able to achieve up to ten percent speedup. In particular, by relying exclusively on the manual implementation, we have improved the performance of one of the most importantand highly optimized-services at Google by $+0.65 \% \pm 0.1 \%$ Requests Per Second (RPS). We have witnessed similar gains throughout the fleet which lead to an overall $1 \%$ performance increase.

\section{References}

[1] ARM. 2020. memcpy-advsimd.S. https://github.com/ARM-software/ optimized-routines/blob/master/string/aarch64/memcpy-advsimd.S

[2] Grant Ayers, Nayana Prasad Nagendra, David I. August, Hyoun Kyu Cho, Svilen Kanev, Christos Kozyrakis, Trivikram Krishnamurthy, Heiner Litz, Tipp Moseley, and Parthasarathy Ranganathan. 2019. AsmDB: Understanding and Mitigating Front-End Stalls in WarehouseScale Computers. In International Symposium on Computer Architecture (ISCA).

[3] Prasanna Balaprakash, Jack Dongarra, Todd Gamblin, Mary Hall, Jeffrey K. Hollingsworth, Boyana Norris, and Richard Vuduc. 2018. Autotuning in High-Performance Computing Applications. Proc. IEEE 106,
11 (2018), 2068-2083. https://doi.org/10.1109/JPROC.2018.2841200

[4] JF Bastien. 2018. Deprecating volatile. http://wg21.link/P1152R0

[5] Eli Bendersky. 2011. Load-time relocation of shared libraries. https:/eli.thegreenplace.net/2011/08/25/load-time-relocation-ofshared-libraries

[6] Eli Bendersky. 2011. Position Independent Code (PIC) in shared libraries. https://eli.thegreenplace.net/2011/11/03/positionindependent-code-pic-in-shared-libraries

[7] Eli Bendersky. 2011. Position Independent Code (PIC) in shared libraries on x64. https://eli.thegreenplace.net/2011/11/11/positionindependent-code-pic-in-shared-libraries-on-x64

[8] Chandler Carruth. 2015. CppCon 2015: Chandler Carruth "Tuning C++: Benchmarks, and CPUs, and Compilers! Oh My!". https://youtu. be/nXaxk27zwlk?t=2441

[9] Guillaume Chatelet. 2019. [llvm-dev] [RFC] [clang/llvm] Allow efficient implementation of libc's memory functions in $\mathrm{C} / \mathrm{C}++$. https://lists. Ilvm.org/pipermail/Ilvm-dev/2019-April/131973.html

[10] Guillaume Chatelet. 2020. automemcpy paper supplementary materials. http://github.com/google-research/automemcpy

[11] Guillaume Chatelet. 2020. Benchmarking llvm-libc's memory functions. https://github.com/Ilvm/llvm-project/blob/main/libc/ benchmarks/RATIONALE.md

[12] Guillaume Chatelet. 2020. [libc-dev] Q\&A and the round table highlights from the virtual dev meeting. https://lists.Ilvm.org/pipermail/ libc-dev/2020-October/000211.html

[13] Guillaume Chatelet. 2020. Libc mem* benchmarking framework. https: //github.com/Ilvm/Ilvm-project/tree/main/libc/benchmarks

[14] Guillaume Chatelet and "other contributors". 2020. Libc string functions. https://github.com/llvm/llvm-project/tree/main/libc/src/string

[15] Dehao Chen, David Xinliang Li, and Tipp Moseley. 2016. AutoFDO: Automatic Feedback-Directed Optimization for Warehouse-Scale Applications. In CGO 2016 Proceedings of the 2016 International Symposium on Code Generation and Optimization. New York, NY, USA, 12-23.

[16] Leonardo de Moura and Nikolaj Bjørner. 2008. Z3: An Efficient SMT Solver. In Tools and Algorithms for the Construction and Analysis of Systems, C. R. Ramakrishnan and Jakob Rehof (Eds.). Springer Berlin Heidelberg, Berlin, Heidelberg, 337-340.

[17] Franz Franchetti, Yevgen Voronenko, and Markus Püschel. 2005. Formal Loop Merging for Signal Transforms. SIGPLAN Not. 40, 6 (June 2005), 315-326. https://doi.org/10.1145/1064978.1065048

[18] M. Frigo and S.G. Johnson. 2005. The Design and Implementation of FFTW3. Proc. IEEE 93, 2 (2005), 216-231. https://doi.org/10.1109/ JPROC.2004.840301

[19] Mathias Gottschlag and Frank Bellosa. 2019. Mechanism to Mitigate AVX-Induced Frequency Reduction. CoRR abs/1901.04982 (2019). arXiv:1901.04982 http://arxiv.org/abs/1901.04982

[20] John L. Henning. 2006. SPEC CPU2006 Benchmark Descriptions. SIGARCH Comput. Archit. News 34, 4 (Sept. 2006), 1-17. https: //doi.org/10.1145/1186736.1186737

[21] ISO/IEC. 2020. ISO International Standard ISO/IEC 9899:202x (E) - Programming Language C [Working draft]. http://www.openstd.org/jtc1/sc22/wg14/www/docs/n2478.pdf

[22] Svilen Kanev, Juan Darago, Kim Hazelwood, Parthasarathy Ranganathan, Tipp Moseley, Gu-Yeon Wei, and David Brooks. 2014. Profiling a warehouse-scale computer. In ISCA '15 Proceedings of the 42nd Annual International Symposium on Computer Architecture. 158-169.

[23] Donald E. Knuth. 1971. Optimum binary search trees. Acta informatica 1, 1 (1971), 14-25.

[24] Vlad Krasnov. 2017. On the dangers of Intel's frequency scaling. https: //blog.cloudflare.com/on-the-dangers-of-intels-frequency-scaling/

[25] Michael Matz and Janothers. 2014. System V Application Binary Interface AMD64 Architecture Processor Supplement (With LP64 and ILP32 Programming Models) Version 1.0. https://gitlab.com/x86psABIs/x86-64-ABI 
[26] Carlos O’Donell. 2017. What is an indirect function (IFUNC)? https: //sourceware.org/glibc/wiki/GNU_IFUNC

[27] Linus Torvalds. 2021. Linux Perf Tool. https://github.com/torvalds/ linux/tree/master/tools/perf

[28] Travis Downs aka BeeOnRope and Arnaud. 2019. Enhanced REP MOVSB for memcpy. https://stackoverflow.com/a/43574756

[29] various contributors. 2021. Glibc memcpy Implementation Strategies. https://sourceware.org/git/?p=glibc.git;a= blob;f=sysdeps $/$ x86_64/multiarch/memmove-vec-unalignederms.S;hb=6e02b3e9327b7dbb063958d2b124b64fcb4bbe3f\#120
[30] R. Whaley and Antoine Petitet. 2005. Minimizing development and maintenance costs in supporting persistently optimized BLAS. Software: Practice and Experience 35 (02 2005), 101-121. https://doi.org/10. 1002/spe.626

[31] Huan Ying, Hao Zhu, Donghui Wang, and Chaohuan Hou. 2013. A novel scheme to generate optimal memcpy assembly code. In 2013 IEEE Third International Conference on Information Science and Technology (ICIST). 594-597. https://doi.org/10.1109/ICIST.2013.6747619 